\title{
Geociências
}

\section{Arsênio na água subterrânea em Ouro Preto e Mariana, Quadrilátero Ferrífero (MG)}

\author{
Ricardo Perobelli Borba \\ Pesquisador Dr. do Instituto Agronômico de Campinas.E-mail: borba@iac.sp.gov.br \\ Bernardino Ribeiro Figueiredo \\ Prof. Dr. do Instituto de Geociências - Unicamp.E-mail: berna@ige.unicamp.br \\ José Adilson Cavalcanti \\ Prof. Dr.do Instituto Politécnico, PUCMINAS.E-mail: adilson@pucpcaldas.br
}

\section{Resumo}

No Quadrilátero Ferrífero (QF), o abastecimento público de água é feito principalmente com a captação de águas das bacias hidrográficas. Entretanto, em alguns locais, há a utilização de água subterrânea, proveniente de nascentes ou mesmo de minas abandonadas, para o abastecimento humano.

As principais fontes naturais de As no QF estão relacionadas às rochas que hospedam depósitos auríferos sulfetados. As fontes antrópicas de As são as pilhas de rejeito, solos e sedimentos contaminados. Em águas coletadas em algumas minas auríferas subterrâneas e nascentes das regiões de Ouro Preto e Mariana, foram encontradas concentrações de As total variando de 2 a $2980 \mu \mathrm{g} / \mathrm{L}$ e de $\mathrm{As}^{3+}$ de 1 até $86 \mu \mathrm{g} / \mathrm{L}$.

Com base nos estudos geológicos e hidrogeoquímicos da ocorrência de As, nas rochas e nas águas subterrâneas, observou-se que as maiores concentrações de As são encontradas nos aqüíferos hospedados em rochas que possuem sulfetos e carbonatos, embora as concentrações dos aqüíferos que possuem somente sulfetos também foram elevadas.

O monitoramento de As na água subterrânea deve ser periódico, independentemente de sua ausência num primeiro momento, nos poços e em outras fontes de captações de águas subterrâneas atualmente em utilização no abastecimento público ou privado.

Palavras-chaves: arsênio, água subterrânea, exposição humana, ouro, Quadrilátero Ferrífero.

\begin{abstract}
In the Iron Quadrangle region the public supply of water is done mainly by the impound of water of watersheds. However, in some places, the population use spring water or ground water from closed mines for human consumption.

The main natural sources of As in the Iron Quadrangle are related to the rocks that contain lode gold deposits. The antropic sources of As are contaminated refuse piles, soil, and sediment. It was found total As concentration varying from 2 to $2,980 \mu \mathrm{g} / \mathrm{L}$ and $A s^{3+}$ from 1 to $86 \mu \mathrm{g} / \mathrm{L}$ in water samples collected in underground gold mines, artesian wells, and springs in Ouro Preto and Mariana counties.

Based on geological and hydrogeochemical studies of the occurrence of As in rocks and groundwater, it was found that the highest As concentrations occurred in aquifers hosted in carbonate sulphide rich rocks.

The As monitoring in underground water should be done periodically, independent of its absence in a first instance, in wells and other groundwater caption sources that are used nowadays for public or private suppliers.
\end{abstract}

Keywords: arsenic, groundwater, human exposure, gold, Quadrilátero Ferrifero. 


\section{Introdução}

No Quadrilátero Ferrífero (QF), o abastecimento público de água é feito principalmente com a captação de águas das bacias hidrográficas do alto rio das Velhas e do rio Doce. Na bacia do rio Doce, no ponto de amostragem localizado em Monsenhor Horta-MG, o rio do Carmo apresentou Índice de Qualidade das Águas ruim no ano 2001. O aumento da vazão no verão provocou uma degradação da qualidade das águas devido ao carreamento da poluição para o rio. Os lançamentos de esgoto in natura pelos municípios de Ouro Preto e Mariana provocaram os altos valores de coliformes fecais e fosfato. As atividades de mineração e garimpo na sub-bacia do Rio do Carmo podem ter sido responsáveis pela ocorrência das elevadas concentrações de mercúrio, além do elevado teor de manganês verificado em suas águas (IGAM, 2002a). Na bacia do rio Doce não há o monitoramento do As.

Nos municípios de Ouro Preto e Mariana o abastecimento público é feito através da captação de água superficial, de nascentes e em Ouro Preto também são utilizadas águas subterrâneas provenientes de antigas minas de ouro. As populações dessas localidades também coletam água de pequenas nascentes nas encostas de morros, principalmente nos distritos distantes da sede do município.

A utilização da água subterrânea no abastecimento público pode vir a ser uma alternativa atraente em virtude da qualidade geralmente superior quando comparada com as águas superficiais, em relação à contaminação por esgotos domésticos e até mesmo industriais, e também por sofrerem uma influência menor da sazonalidade pluviométrica que pode restringir o volume de água captado superficialmente. Entretanto em alguns locais do QF existe a possibilidade da ocorrência de As nas águas subterrâneas, o que torna estas águas impróprias para o consumo humano sem um tratamento específico para a remoção do As.

Nesse trabalho é realizado um estudo hidrogeoquímico com ênfase no As, incluindo a especiação de As inorgâni- co, das águas subterrâneas de aqüíferos, sob influência de rochas portadoras de mineralizações auríferas, de alguns pontos das regiões de Ouro Preto e Mariana.

\subsection{O As e a saúde humana}

Em águas naturais, o arsênio está presente principalmente na forma de compostos inorgânicos, onde possui as valências $3+$ e 5+ (Thornton \& Farago,1997). A toxicidade das diversas espécies de arsênio decresce na seguinte ordem: compostos de $\mathrm{As}^{3+}$ inorgânico > compostos de $\mathrm{As}^{5+}$ inorgânico $>$ compostos de $\mathrm{As}^{3+}$ orgânico $>$ compostos de $\mathrm{As}^{5+}$ orgânico (USEPA, 2000). Em termos de intensidade, o $\mathrm{As}^{3+}$ inorgânico é 60 vezes mais tóxico que o $\mathrm{As}^{5+}$ inorgânico (Mabuchi et al., 1979).

Os principais modos de intoxicação por arsênio ocorrem via consumo de águas poluídas e por ingestão de solos contaminados (USEPA, 2000). A intoxicação por arsênio pode resultar em efeitos tóxicos, agudos ou crônicos, relativos a exposições curtas ou longas, respectivamente, ocasionando diferentes patologias.

Os efeitos carcinogênicos da intoxicação por As estão associados à exposição crônica por vários anos. Os tipos de câncer associados à exposição crônica são o câncer de pele, pulmão (inalação), próstata, bexiga, rim e fígado (NRC, 1999).

A intoxicação aguda e crônica por As provoca diversas outras patologias não cancerígenas à saúde humana: cutâneas (hiperpigmentação, hiperqueratose); gastrintestinais (diarréia, hemorragias gastrintestinais); cardiovasculares (arritmias cardíacas, hipotensão e falha congestiva no coração, problemas no sistema circulatório vascular levando à gangrena); hematológicos (anemia), pulmonares (fibrose); neurológicos (dores de cabeça, confusão mental, coma); endocrinológicos (problemas no metabolismo de carboidratos e respiração celular); reprodutivos e de desenvolvimento como abortos espontâneos e fetos com baixo peso (USEPA,2000; WHO,2001a).
Em 1984 a Organização Mundial de Saúde (WHO) sugeriu que as águas potáveis deveriam ter no máximo $50 \mu \mathrm{g} / \mathrm{L}$ de As . Com base em novas evidências toxicológicas, a WHO rebaixou em 1993 a concentração máxima de As em água para $10 \mu \mathrm{g} / \mathrm{L}$ (WHO, 2001b). Atualmente no Brasil o limite máximo permitido é de $10 \mu \mathrm{g} / \mathrm{L}$ (Funasa, 2001).

Em Bangladesh e West Bengal (Índia), ocorre o pior caso de contaminação de água subterrânea e humana por As do mundo, afetando cerca de 42 milhões de pessoas (Smedley \& Kinniburgh, 2002).

A contaminação humana por As no QF, nas regiões de Nova Lima e Brumal, foi pesquisada por Matschullat et al. (2000) através de um estudo da presença de As em urina de crianças de 7 a 12 anos.Entre a população infantil avaliada foram encontradas concentrações de As entre 2 e $106 \mu \mathrm{g} / \mathrm{L}$ nas amostras de urina, sendo que $22 \%$ das crianças apresentaram concentrações superiores a $40 \mu \mathrm{g} /$ L para as quais efeitos adversos não podem ser excluídos.

\subsection{A distribuição do As nas rochas, sedimentos e águas superficiais do QF}

O QF é uma região com inúmeras mineralizações auríferas sulfetadas. Quatro unidades litoestratigráficas principais compõem o QF: os complexos metamórficos granito-gnaissico, uma seqüência greenstone belt arqueana denominada de Supergrupo Rio das Velhas, os metassedimentos e metavulcânicas proterozóicas do Supergrupo Minas, e os metassedimentos do Grupo Itacolomi (Dorr, 1969).

A distribuição geográfica do As nas rochas do QF que se conhece atualmente guarda uma associação importante com as rochas auríferas sulfetadas. Os principais depósitos auríferos do QF estão concentrados nas porções norte e sul, totalizando 66 minas, a maioria delas atualmente fechadas (Ladeira,1988). No mapa de Pinto (1996) podem ser obser- 
vada, além das principais minas, a existência de inúmeras minas de menor porte e de garimpos. A maioria dos depósitos auríferos, principalmente os da porção norte do QF, está hospedada nas rochas do Supergrupo Rio das Velhas. Além desses depósitos, também há a ocorrência de mineralizações situadas nas rochas do Supergrupo Minas, como os depósitos da região de Ouro Preto Mariana e os depósitos de Au do tipo Jacutinga, que ocorrem nas minas de ferro da Formação Cauê (Gongo Soco, Morro Água Quente, Cata Preta, Maquine e Itabira, Conceição, Periquito, Cauê).

Estudos anteriores sobre a presença de As em solos, sedimentos e água no QF foram realizados por Eleutério (1997), Rawlins et al. (1997), Deschamps et al. (2002), Pimentel et al. (2003) e Borba et al. $(2000,2003 a)$. Nos sedimentos e nas águas superficiais do QF, Borba et al. (2003a) constataram que os maiores teores de As também estão localizados nas proximidades das mineralizações auríferas, onde as concentrações de As podem alcançar até $4500 \mathrm{mg} / \mathrm{kg}$ nos sedimentos e $350 \mu \mathrm{g} / \mathrm{L}$ na água. Nos sedimentos do QF, o As está associado a óxidos de ferro e sulfetos. As origens do As nos sedimentos e nas águas superficiais se devem à oxidação natural das rochas e principalmente à atividade antrópica, que se deu por meio do lançamento dos rejeitos da mineração nas drenagens. Segundo estimativas de Borba et al. (2000), a atividade antrópica provocou o lançamento nos rios do QF de, pelo menos, 390.000t de As durante 300 anos de mineração.

Mesmo em outras drenagens, situadas em cotas mais elevadas e que cortam as rochas do Supergrupo Minas, o As foi encontrado nos sedimentos sem que houvesse no local o aporte de sedimentos provenientes da erosão de rochas mineralizadas dos depósitos auríferos acima citados. Isto sugere que a distribuição do As nas rochas do QF não estaria restrita apenas àquelas rochas que possuem mineralizações auríferas (Borba et al., 2003a).

\section{Material e métodos}

As amostras de água foram coletadas em julho de 2000. Em cada ponto de coleta foram medidos in situ o $\mathrm{pH}, \mathrm{Eh}$, a condutividade e a temperatura, e coletadas amostras de água filtradas e não filtradas. Foram coletadas quatro amostras de água. Três dessas amostras foram filtradas através de uma membrana de acetato de celulose de $0,45 \mu \mathrm{m}$ (Millipore ${ }^{\circledR}$ ). Uma das amostras foi mantida ao natural para análise dos ânions, outras duas foram aciduladas com $0,2 \%$ de $\mathrm{HNO}_{3}$ P.A., para análise dos cátions e especiação de As. Nas amostras filtradas, os cátions e os ânions foram determinados por ICPAES e por cromatógrafo de íons, respectivamente. A amostra não filtrada, de $500 \mathrm{ml}$ foi utilizada para a determinação da alcalinidade do bicarbonato por titulação, usando ácido sulfúrico $(0,16 \mathrm{~N})$ e indicador verde de bromocresol.

A determinação de As total foi feita por HG-AAS. A especiação inorgânica do As foi feita pelo método desenvolvido por Borba et al.(2003b) utilizando o HG-AAS.

\section{Resultados e Discussões}

As amostras de água foram coletadas em minas subterrâneas, poços artesianos e nascentes das regiões de Ouro Preto e Mariana, um dos distritos auríferos do QF (Figura 1, Tabela 1). Os parâmetros físico-químicos e os principais cátions e ânions determinados nessas águas, salvo raras exceções, possuem valores compatíveis ao consumo humano segundo a Portaria 1.469 da FUNASA de dezembro de 2000 (Tabela 1).

As concentrações de As total determinadas nas amostras de água variaram de 2 a $2980 \mu \mathrm{g} / \mathrm{L}$, sendo que, na maioria das amostras, os valores são superiores ao valor máximo permitido para consumo humano (Tabela 1), que é de $10 \mu \mathrm{g} / \mathrm{L}$ de As (FUNASA, 2001). Em algumas amostras, foi feita a especiação inorgânica do As, tendo sido encontrado o $\mathrm{As}^{3+}$, a espécie mais tóxica de As, em concentrações de até $86 \mu \mathrm{g} / \mathrm{L}$ de $\mathrm{As}^{3+}$. As amostras de água com concentrações de As impróprias para consumo humano foram encontradas na mina de Passagem, em Passagem de Mariana (município de Mariana) e em minas e nascentes de Ouro Preto, com exceções das amostras M-4 e M-5, da mina de Passagem e de uma nascente em Passagem de Mariana, que possuem concentrações de As inferiores a $10 \mu \mathrm{g} / \mathrm{L}$. É importante ressaltar que as concentrações de As determinadas nas amostras de água são representativas do momento da amostragem e podem sofrer variações em seus valores, para mais ou para menos, ao longo do tempo.

Um aspecto importante sobre a presença do As na água subterrânea é o modo errático de sua ocorrência. Concentrações discrepantes de As na água foram observadas em locais próximos ou num mesmo local, conforme pode ser observado em amostras coletadas em Ouro Preto (O-1 e O-2) e na mina de Passagem, respectivamente. Em Ouro Preto, em amostras que foram coletadas em minas situadas aproximadamente a $200 \mathrm{~m}$ de distância entre si, os valores das concentrações de As encontradas foram de 260 e $24 \mu \mathrm{g} / \mathrm{L}$, enquanto que, na mina de Passagem, as amostras M-3 e M-4, que foram coletadas em pontos distanciados de algumas dezenas de metros, possuem concentrações de As de 4 e $2980 \mu \mathrm{g} / \mathrm{L}$ (Tabela 1).

As maiores concentrações de As na água foram obtidas junto a rochas sulfetadas ricas em carbonatos, como as da Mina da Passagem. Em contraposição, as rochas mineralizadas das Minas de Ouro Preto, pobres em carbonatos, apresentaram as concentrações menores de As (Borba \& Figueiredo in press). Nas demais amostras de água, com concentrações de As inferiores a $10 \mu \mathrm{g} / \mathrm{L}$, coletadas na mina de Passagem e na nascente em Passagem de Mariana, provavelmente não houve o contato da água subterrânea com rochas/solos que possuíssem minerais primários ou secundários de As.

A origem do As encontrado nas amostras de água subterrânea se deve 


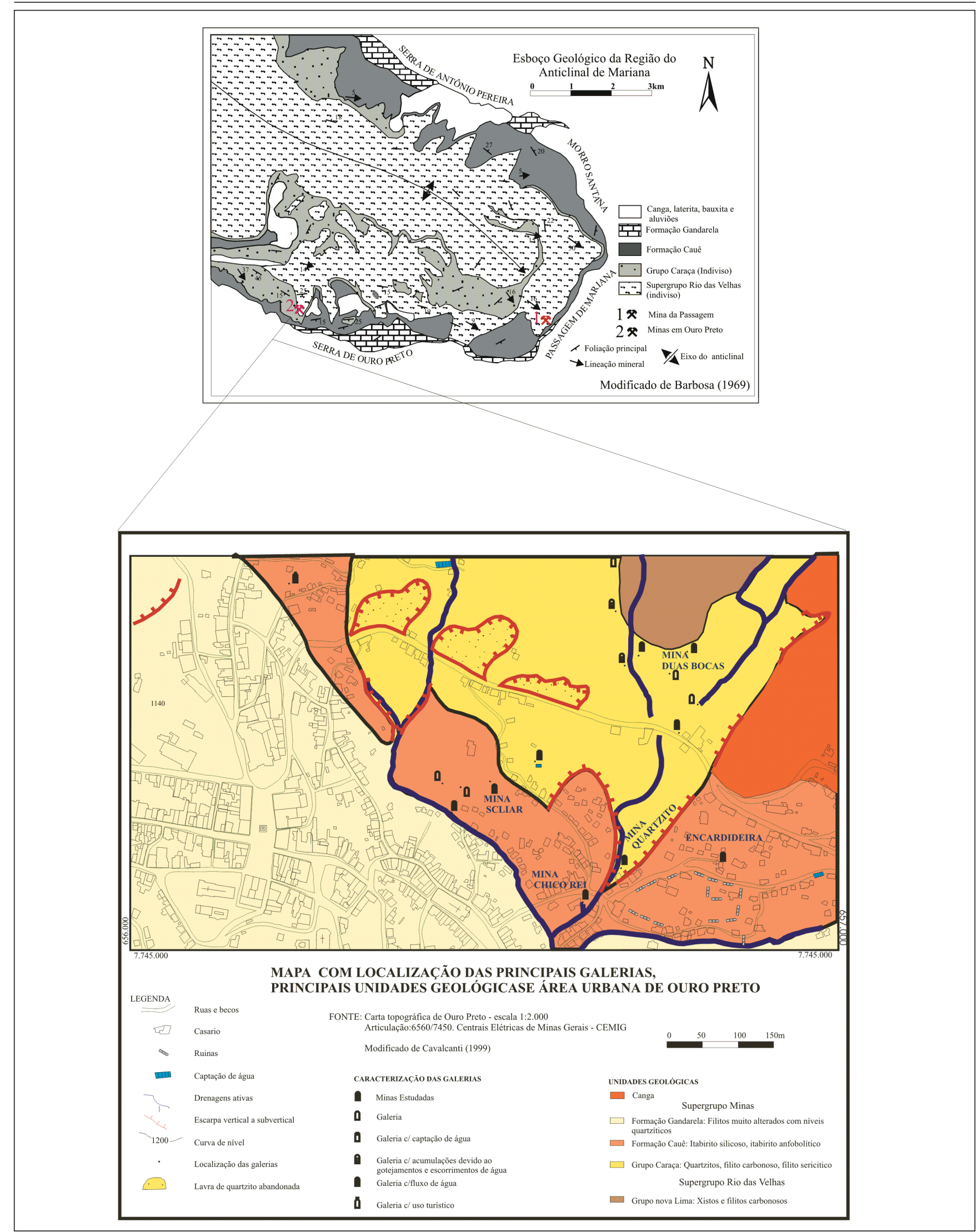

Figura 1 - Mapa geológico simplificado do Anticlinal de Mariana com detalhe da área urbana de Ouro Preto indicando a localização dos principais locais de coleta de amostras de água. 
Ricardo Perobelli Borba et al.

Tabela 1 - Composição química das amostras de água subterrânea.

\begin{tabular}{|c|c|c|c|c|c|c|c|c|c|c|c|c|c|c|}
\hline & \multirow[b]{2}{*}{ LD } & \multicolumn{5}{|c|}{ Passagem de Mariana } & \multicolumn{7}{|c|}{ Ouro Preto } & \multirow{2}{*}{$\begin{array}{c}\text { FUNASA } \\
\text { P } 1469 \\
\end{array}$} \\
\hline & & M-1 & M-2 & M-3 & M-4 & M-5 & $0-1$ & $0-2$ & $0-3$ & $0-4$ & $0-5$ & $0-6$ & $0-7$ & \\
\hline $\mathrm{pH}$ & & 8 & 8 & 7 & 7 & 6 & 7 & 7 & 6 & 6 & 6 & 7 & 6 & $6-9,5$ \\
\hline Eh $(\mathrm{mV})$ & & 389 & 358 & 360 & 380 & 300 & 430 & 481 & 515 & 330 & 515 & 505 & 545 & nd \\
\hline Cond $(\mu \mathrm{S} / \mathrm{cm})$ & & 372 & 253 & 180 & 371 & 93 & 70 & 17 & 51 & 15 & 0,1 & 0,1 & 0,1 & nd \\
\hline $\mathrm{T}\left({ }^{\circ} \mathrm{C}\right)$ & & 20 & 23 & 21 & 20 & 21 & 19 & 15,3 & 15 & 18 & 17 & 18 & 19 & nd \\
\hline Dureza & & 192 & 91 & 110 & 240 & 27 & 31 & 9 & 22 & 10 & 21 & 20 & 67 & 500 \\
\hline $\mathrm{Ca}$ & 0,05 & 41 & 22 & 21 & 47 & 7 & 6 & 2 & 3 & 2 & 1 & 1,4 & 4 & nd \\
\hline $\mathrm{Mg}$ & 0,3 & 22 & 12 & 14 & 30 & 2 & 4 & 1 & 2 & 1 & 0,5 & 0,6 & 1 & nd \\
\hline $\mathrm{Na}$ & 0,1 & 2 & 2 & 0,5 & 3 & 5 & 0,5 & 0,6 & 2 & 0,2 & 5 & 3,6 & 8 & 200 \\
\hline $\mathrm{K}$ & 0,1 & 3 & 3 & 1 & 1 & 3 & 1 & 0,4 & 1 & 0,2 & 2 & 1,8 & 3 & nd \\
\hline $\mathrm{HCO}_{3}$ & & 121 & 55 & 74 & 234 & 5 & 12 & 8 & 4 & 12 & 8 & 9 & 5 & nd \\
\hline $\mathrm{Cl}$ & 0,1 & 11 & 2 & 1 & 4 & 7 & 3 & 1 & 4 & 1 & 8 & 6 & 12 & 250 \\
\hline $\mathrm{SO}_{4}$ & 0,2 & 52 & 33 & 38 & 10 & 11 & 19 & 2 & 10 & 1 & 1 & 2 & 7 & 250 \\
\hline $\mathrm{NO}_{3}$ & 0,2 & 4 & 2 & 1 & 0,3 & 18 & 3 & 2 & 5 & 1 & 14 & 13 & 65 & 44 \\
\hline $\mathrm{F}$ & 0,005 & 0,1 & 0,2 & 0,1 & 0,1 & 0,1 & $<\mathrm{LD}$ & $<\mathrm{LD}$ & $<\mathrm{LD}$ & $<\mathrm{LD}$ & $<L D$ & $<\mathrm{LD}$ & $<\mathrm{LD}$ & 1,5 \\
\hline $\mathrm{PO}_{4}$ & 0,05 & 0,1 & $<\mathrm{LD}$ & $<\mathrm{LD}$ & $<L D$ & $<L D$ & 0,2 & $<\mathrm{LD}$ & $<\mathrm{LD}$ & $<\mathrm{LD}$ & $<L D$ & $<\mathrm{LD}$ & $<\mathrm{LD}$ & nd \\
\hline $\mathrm{Mn}$ & 0,001 & $<\mathrm{LD}$ & $<\mathrm{LD}$ & 0,01 & 0,01 & $<L D$ & 0,2 & $<\mathrm{LD}$ & $<\mathrm{LD}$ & $<\mathrm{LD}$ & $<L D$ & $<\mathrm{LD}$ & $<\mathrm{LD}$ & 0,1 \\
\hline $\mathrm{Fe}$ & 0,006 & 0,1 & 0,1 & 0,1 & 0,1 & $<L D$ & 0,1 & $<\mathrm{LD}$ & $<L D$ & $<L D$ & $<\mathrm{LD}$ & $<\mathrm{LD}$ & $<\mathrm{LD}$ & 0,3 \\
\hline AsTotal & 0,8 & 830 & 1760 & 2980 & 4 & 2 & 260 & 24 & 32 & 12 & 12 & 22 & 62 & 10 \\
\hline $\mathrm{As}^{3+}$ & & 35 & 59 & 86 & 1 & - & 12 & - & - & - & - & - & - & - \\
\hline $\mathrm{As}^{5+}$ & & 795 & 1701 & 2894 & 3 & - & 48 & - & - & - & - & - & - & - \\
\hline
\end{tabular}

Localização das Amostras

Mina de Passagem: M-1 - adit lateral, M-2 - lago interno, M-3 - gotejamento Gal. Sindicato, M-4 - gotejamento nível 120; M-5 - Nascente em residência no Boqueirão, em Passagem de Mariana.

Minas em Ouro Preto (região das Lajes), amostras coletadas em gotejamentos: O-1 - Chico Rei, O-2 -

Quartzito, O-3 - Scliar; amostras coletadas na boca da mina, O-4 - Encardideira, O-5 - Piedade de Baixo, O-6 -

Piedade de Cima, O-7 - Bica do Padre Faria (nascente)

Concentrações em mg/L, exceto para o As, em $\mu \mathrm{g} / \mathrm{L}$. LD - Limite de Detecção; nd - não definido.

ao processo de oxidação natural da arsenopirita e da pirita encontradas nas rochas auríferas sulfetadas. Segundo Borba e Figueiredo (in press), no processo de oxidação desses sulfetos, promovido pelo intemperismo, há a formação de escorodita $\left(\mathrm{FeAsO}_{4} 2 \mathrm{H}_{2} \mathrm{O}\right)$ ou de arsenato de ferro com baixa cristalinida- de, e a liberação do As em solução para a água subterrânea. Posteriormente, devido à elevação do $\mathrm{pH}$ do meio, há a dissolução incongruente da escorodita ou do arsenato de ferro com baixa cristalinidade previamente formados, o que resulta na formação de goethita e liberação do As em solução para a água sub- terrânea. Os processos de oxidação de sulfetos e dissolução incongruente dos minerais secundários acima descritos também foram observados em exposições superficiais de rochas mineralizadas nas regiões das Lajes, em Ouro Preto, na pedreira localizada em Antônio Pereira, em Raposos e em Caeté. Nesses 
casos, o As solubilizado pode ser tanto transportado pelas chuvas e alcançar as drenagens como se infiltrar no solo com a água, alcançando desse modo a água subterrânea (Borba \& Figueiredo in press).

\section{Considerações Finais}

Nas amostras de água subterrânea de Ouro Preto e Mariana, foi constatado que as maiores concentrações de As ocorreram em locais com a presença de sulfetos e carbonatos nas rochas. Nas rochas que só possuíam sulfetos, as concentrações, embora elevadas, foram inferiores às das rochas com carbonato.

O carbonato, ao reagir com as soluções ácidas decorrentes da oxidação dos sulfetos, é dissolvido, proporcionando o aumento do $\mathrm{pH}$ da solução e o favorecimento da solubilização dos compostos de As, conforme foi observado na mina de Passagem, que possui carbonato em suas rochas. Por analogia, a mitigação de impactos ambientais causados pela drenagem ácida proveniente da atividade da mineração e por passivos ambientais como antigos rejeitos de mineração, através da adição de carbonatos para a neutralização da acidez da solução, pode favorecer o aumento da mobilidade do As, caso ele esteja presente nos solos, sedimentos ou rejeitos remediados.

As grandes variações das concentrações de As apresentadas em locais próximos, como observado em Ouro Preto, ou num mesmo local, como na mina de Passagem, indicam que a heterogeneidade hidrogeoquímica da água subterrânea na área estudada pode ocorrer em distâncias que variam de centenas ou de apenas alguns metros.

A situação observada de contaminação natural de água subterrânea por As nos pontos estudados em Ouro Preto e Mariana pode se repetir em outros locais do QF que possuam contexto geológico semelhante, ou seja, rochas contendo mineralizações aurí- feras ou a presença de sulfetos em rochas, solos e sedimentos, tanto na bacia do rio Doce como na bacia do alto rio das Velhas. Vale salientar que, na bacia do alto rio das Velhas, o As é encontrado nas águas superficiais, monitoradas pelo Projeto Águas de Minas, acima dos limites permitidos para o consumo humano em inúmeros pontos da bacia (IGAM, 2002b).

Em virtude da presença de As nos sedimentos dos rios, nos solos, nas rochas, nas águas superficiais e subterrâneas em vários pontos do $\mathrm{QF}$, deve haver um monitoramento constante para a concentração do As nas águas utilizadas no abastecimento público nas áreas de risco, pois os parâmetros de qualidade de água normalmente avaliados não levam em consideração a presença de As e os tratamentos de adição de cloro e flúor não removem o As da água. Em Ouro Preto, especificamente, a coleta de água na mina de Piedade, para abastecimento público, deve ser monitorada para as concentrações de As, uma vez que a amostra coletada apresentou concentração de As um pouco acima do limite de potabilidade que é de $[\mathrm{As}]=10 \mu \mathrm{g} / \mathrm{L}$.

No caso da utilização de águas coletadas em poços artesianos, rasos ou profundos, deve-se ter atenção especial, uma vez que pode haver a contaminação de poços inicialmente isentos de As ao longo do tempo devido: (i) à oxidação de sulfetos presentes no aqüífero, como descrito por Schreiber et al.(2000); (ii) às mudanças no regime de fluxo da água nos aqüíferos fraturados e captação de águas contaminadas, pois, como observado nas minas do Chico Rei e Quartzito e na mina de Passagem, é possível se encontrar água com concentrações de As relativamente baixas próximas a locais contendo água com elevados teores de As, que podem, eventualmente, ser capturadas pelo bombeamento do poço e (iii) à liberação de As para as águas subterrâneas dos aqüíferos aluvionares pelos sedimentos que contêm As, como ocorrido em Bangladesh e West Bengal (Índia).

\section{Agradecimentos}

Esse estudo foi realizado com suporte financeiro da FAPESP (processos 97/14446-6 e 96/7839-9). Os autores são gratos aos srs. Walter Rodrigues e Roberto Rodrigues (Cia Mina de Passagem) pela permissão ao acesso e apoio à realização da pesquisa na mina da Passagem, ao Teinha pelo acompanhamento nos trabalhos na mina de Passagem e à dona Mariazinha e seus filhos pelo acesso à mina do Chico Rei.

\section{Referências bibliográficas}

BARBOSA, A.L.M. Geológic map of the Ouro Preto, Mariana, Antônio Pereira e São Bartolomeu quadrangles, Minas Gerais, Brazil. Washington, DNPM/USGS, 1969. U.S. Geol. Surv. Prof. Paper, 641-A. Plates 7, 8, 9, 10 .

BORBA, R.P., FIGUEIREDO, B.F. A influência das condições geoquímicas na oxidação da arsenopirita e na mobilidade do arsênio em ambientes superficiais tropicais. Revista Brasileira de Geociências, (in press).

BORBA, R.P., FIGUEIREDO, B.R., MATSCHULLAT, J. Geochemical distribution of arsenic in waters, sediments and weathered gold mineralized rocks from Iron Quadrangle, Brazil. Environmental Geology, v.4, p.39-52, 2003 a.

BORBA, R.P., FIGUEIREDO, B.F., ZAMBELLO, F. Método quantitativo de análise do $\mathrm{As}_{\text {Total }}$, especiação do As em amostras de água: aplicação no monitoramento ambiental de As em água. In: CONG. BRAS. GEOQ., 9, 2003. Belém, Anais... Belém: SBGq, 2003b, p. 313-315. 2003.

BORBA, R.P., FIGUEIREDO, B.R., RAWLLINS, B.G., MATCHULLAT, J. Arsenic in water and sediment in the Iron Quadrangle, Minas Gerais state, Brasil. Revista Brasileira de Geociências, v.30, n.3, p.554-557, 2000.

CAVALCANTI, J.A.D. Mineralização aurífera de Lajes-Antônio Dias, Ouro Preto - MG: controles lito-estratigráficos e estruturais. Instituto de Geociências, Unicamp, 1999. 108p. (Dissertação de mestrado).

DESCHAMPS, E., CIMINELLI, V.S.T., LANGE, F.T., MATSCHULLAT, J., RAUE, B., SCHMIDT, H. Soil and Sediment Geochemistry of the Iron Quadrangle, Brazil: The Case of Arsenic. $\boldsymbol{J}$ Soils \& Sediments, v.2, n.4, p.216-222, 2002.

DORR II, J. V. N. Physiographic, stratigraphic and structural development of the QF, Brazil. Prof. Paper 641-A. Washington, DNPM/USGS, 1969. 109p. 
Ricardo Perobelli Borba et al.

ELEUTERIO, L. Diagnóstico da situação ambiental da cabeceira da bacia do rio Doce, no âmbito das contaminações por metais pesados em sedimentos de fundo. Depto. de Geologia, Escola de Minas, Universidade Federal de Ouro Preto, 1997. 163p. (Dissertação de mestrado).

FUNASA 2001. Portaria $\mathrm{n}^{0} 1.469 / 2000$, de 29 de dezembro de 2000: aprova o controle e vigilância da qualidade da água para consumo humano e seu padrão de potabilidade. Brasília: Fundação Nacional de Saúde, 2001. 32 p.

IGAM. Relatório de monitoramento das águas superficiais na bacia do rio Doce em 2001. Projeto Qualidade das águas superficiais no Estado de Minas em 2001. Belo Horizonte: Instituto Mineiro de Gestão das Águas (IGAM), 2002a. 205p.

IGAM. Relatório de monitoramento das águas superficiais na bacia do rio São Francisco em 2001, sub-bacia do rio das Velhas. Projeto Qualidade das águas superficiais no Estado de Minas em 2001. Belo Horizonte: Instituto Mineiro de Gestão das Águas (IGAM), 2002b. 205p.

LADEIRA, E.A. Metalogenia dos depósitos de ouro do Quadrilátero Ferrífero, Minas Gerais, Brasil. In: SCHOBBENHAUS,C. \& COELHO, C.E.S. (eds.) Principais Depósitos Minerais do Brasil. Brasília: DNPM/CVRD, 1988. v.3, p. 301-375.
MABUCHI, K., LILIENFELD, A.M., SNELL, L.M. Lung-cancer among pesticide workers exposed to inorganic arsenicals. Arch. Environ. Health, v.34, p.312-320, 1979.

MATSCHULLAT, J., BORBA, R.P., DESCHAMPS, E., FIGUEIREDO, B.F., GABRIO, T., SCHWENK, M. Human and environmental contamination in the Iron Quadrangle, Brazil. Applied Geochemistry, v.15, p.181-190, 2000.

NRC (National Research Council. Arsenic in drinking water. Washington, DC. National Academic Press, 1999. 310p

PIMENTEL, H.S., LENA, J.C., NALINI JR, H.A. Studies of water quality in the Ouro Preto region, Minas Gerais, Brazil: The release of arsenic to the hydrological system. Environmental Geology, v.43, p.725-730, 2003.

PINTO, C.P. Projeto rio das Velhas. Belo Horizonte: CPRM, 1996. 121p

RAWLINS, B.G., WILLIAMS, T.M., BREWARD, N., FERPOZZI, L., FIGUEIREDO, B.F., BORBA, R.P. Preliminary investigation of mining-related arsenic contamination in the provinces of Mendoza and San Juan (Argentina) and Minas Gerais State (Brazil). London: British Geological Survey, 1997. 25p. (Technical Report WC /97/60).

SCHREIBER, M.J., SIMO, J., FREIBERG, P. Stratigraphic and geochemical controls on naturally ocurring arsenic in groundwater, eastern Wisconsin, USA. Hydrogeology Journal, v.8, p.161-176,2000

SMEDLEY, P.L., KINNIBURGH, D.G. A review of the source,behaviour and distribution of arsenic in natural waters Source and behaviour of arsenic in natural waters. Applied Geochemistry, v.17, p.517$568,2002$.

THORNTON, I., FARAGO, M. The geochemistry of arsenic. In: C.O. ABERNATHY; R.L. CALDERON, W.R. CHAPPELL. (eds.) Arsenic Exposure and health effects. New York: Chapman \& Hall, 1997. cap. 1, p.1-16.

USEPA. National Primary Drinking Water Regulations, Arsenic and Clarifications to Compliance and New Source Contaminants Monitoring; Proposed Rule. Federal Register, v. 65, n. 121, June 22, 2000, p.38888-38983.

WHO. United Nations Synthesis Report on Arsenic in Drinking Water, Geneva, World Health Organization. 2001a (http:// www.who.int/water sanitation health/ Arsenic/ArsenicUNReptoc.htm).

WHO. ARSENIC IN DRINKING WATER. 2001b. Fact Sheet No 210, http:// www.who.int/inf-fs/en/fact210.html.

Artigo recebido em 16/06/2003 e aprovado em 12/04/2004.

\title{
REM - Revista Escola de Minas 68 anos divulgando CIÊNCIA.
}

\author{
$\star \star \star * * *$
}

\section{REM: a mais antiga revista técnico- científica do setor mínero-metalúrgico.}

\author{
$* * * * * *$
}

\section{Assine e Publique}

$* * * * * *$ 